\title{
Clinicopathologic and ultrastructural findings of hereditary nephritis; a 16-year single center survey in Iran
}

\author{
Seyed Mohammad Owji ${ }^{\circledR}$, Hadi Raeisi Shahraki ${ }^{{ }^{\circledR}}{ }^{\mathbb{D}}$, Naser Pajouhi ${ }^{{ }^{\circledR}}$, Seyed Hossein Owji ${ }^{\circledR}$, Farshad \\ Dehghani $^{4^{*} \mathbb{D}}$ \\ ${ }^{1}$ Department of Pathology, School of Medicine, Shiraz University of Medical Sciences, Shiraz, Iran \\ ${ }^{2}$ Department of Epidemiology and Biostatistics, School of Health, Shahrekord University of Medical Sciences, Shahrekord, Iran \\ ${ }^{3}$ Department of Physiology, Faculty of Medicine, Lorestan University of Medical Sciences, Khorramabad, Iran \\ ${ }^{4}$ Student Research Committee, Shiraz University of Medical Sciences, Shiraz, Iran
}

\section{A R T I C L E I N F O}

\section{Article Type:}

Original

\section{Article History:}

Received: 15 October 2019

Accepted: 27 December 2019

Published online: 20 January 2020

\section{Keywords:}

Alport syndrome

Fabry disease

Hereditary nephritis

Kidney

Electron microscopy

\begin{abstract}
A B S T R A C T
Introduction: Hereditary nephritis is an umbrella term for a group of congenital childhood diseases including but not limited to Alport syndrome, thin basement membrane disease, and Fabry disease.

Objectives: The purpose of this study was a clinicopathologic investigation of Alport syndrome, thin basement membrane disease, and Fabry disease with a focus on the role of electron microscopy and toluidine blue staining in diagnosis.

Patients and Methods: In this cross-sectional study, we investigated kidney biopsies with a final diagnosis of either Alport syndrome, thin basement membrane disease or Fabry disease from 2001 to 2016. Electron microscopy and light microscopy were done and the clinical and paraclinical data were extracted from the patients' medical charts. Electron microscopy role was assessed in terms of necessary, helpful or non-necessary, while correlations between clinical and para-clinical data were determined using appropriate statistical tests.

Results: Among the 2865 kidney biopsies, there were 22 patients of hereditary nephritis including $15(0.52 \%)$ Alport syndrome, $5(0.17 \%)$ thin basement membrane disease and 2 (0.07\%) Fabry disease diagnosed by electron microscopy. Electron microscopy was essential for the diagnosis of 19 (86.4\%) cases, helpful for 3(13.6\%) and there was no case for which electron microscopy was non-necessary. The patients' mean age was $16.1 \pm 9.0$ years. The most common finding in Alport syndrome was proteinuria (86.7\%) followed by hematuria (60.0\%). Conclusion: Considering the rate of misdiagnosis of hereditary nephritis using light microscopy and clinical findings alone, electron microscopy study and toluidine blue staining has an essential role in the precise diagnosis in these patients. With regard to the progressive nature of these diseases, prompt diagnosis using electron microscopy is pertinent for therapeutic decisions.
\end{abstract}

Implication for health policy/practice/research/medical education:

This study provides essential tools for clinicians and nephropathologists to help them decide the best approach to the diagnosis of hereditary nephritis as well as for policymakers to determine the necessity of the diagnostic tests and to optimally allocate the available resources to each.

Please cite this paper as: Owji SM, Raeisi Shahraki H, Pajouhi N, Owji SH, Dehghani F. Clinicopathologic and ultrastructural findings of hereditary nephritis; a 16-year single center survey in Iran. J Renal Inj Prev. 2020; 9(2): e17. doi: 10.34172/jrip.2020.17.

\section{Introduction}

The glomerular basement membrane is a specific form of basement membrane that is comprised of four components, type IV collagen being one of them. The importance of the glomerular basement membrane is shown in hereditary glomerular diseases linked to structural anomalies of its components, for example, type IV collagen in Alport syndrome and thin basement membrane disease ( familial benign hematuria), and laminin in Pierson syndrome (1).

A renal biopsy is an important tool for the evaluation of kidney disease. Renal pathologists, with the aid of light microscopy, immunofluorescence microscopy, 
and electron microscopy, reach a final diagnosis in these patients who tend to have overlapping clinical manifestations. The specimen should be divided for light microscopy, immunofluorescence, and electron microscopy (2).

Hereditary glomerular diseases are categorized into several groups including but not limited to; Alport syndrome, thin basement membrane disease, Fabry disease, Nail-Patella syndrome, collagen type III glomerulopathy and fibronectin glomerulopathy $(2,3)$.

Alport syndrome is usually an x-linked, inherited type IV collagen disorder manifested by progressive renal failure and associated problems (like hearing loss and eye involvement) affecting approximately $2.5 \%$ of children and $0.3 \%$ of adults with end-stage renal disease which has characteristic thinning and thickening of glomerular basement membrane with lamina densa splitting in electron microscopy. Thin basement membrane disease is a usually benign, familial hematuria affecting approximately $1 \%$ of the population and characterized by uniform thinning of glomerular basement membrane in electron microscopy. Fabry disease, estimated to range from one in 40000 to one in 117000 live male births. It is a rare hereditary nephritis which is caused by a deficiency of alpha-galactosidase A, resulting in multiorgan involvement and progressive renal failure. Fabry disease has characteristic inclusion bodies "zebra bodies" in electron microscopy (2-6).

For several years, a great effort has been devoted to the study of the pathogenesis, clinical manifestations, and diagnosis of hereditary nephritis in the international scene.

\section{Objectives}

In line with the previous studies, and considering the clinical and pathologic heterogeneity of these diseases and lack of a minimal ultrastructural study of this scale of hereditary nephritis in Iran, we aimed to investigate the clinicopathologic features of hereditary nephritis in this study comprising of Alport syndrome, thin basement membrane disease, and Fabry disease in a 16-year period (2001-2016) with a focus on the role of electron microscopy in the final diagnosis.

\section{Patients and Methods}

In this cross-sectional single-center study, we investigated 2865 kidney biopsies sent to the electron microscopy unit (affiliated to the medical school of Shiraz University of Medical sciences) from 2001 to 2016. All patients diagnosed with Alport syndrome, thin basement membrane disease, and Fabry disease were included in this study. Samples with insufficient kidney tissue and glomeruli were excluded. Finally, 22 patients $(0.77 \%$ of all biopsies) were enrolled in the study.

The fresh renal biopsy tissues were cut into pieces and processed for light microscopy and electron microscopy. For light microscopy, after fixing in formalin and embedding in paraffin, 3-5 $\mu \mathrm{m}$ serial sections were stained with hematoxylin and eosin ( $\mathrm{H} \& \mathrm{E})$, periodic Acid-Schiff, Jones methenamine silver, and Masson's trichrome.

A fresh sample was cut into small sections and processed routinely for electron microscopy. A set of 1-2 $\mu$ sections were taken from resin blocks and stained with toluidine blue for detecting glomeruli under the light microscopy. Then, the $90 \mathrm{~nm}$ sections were put on the copper grid and double-stained with uranyl acetate and lead citrate. The grid was evaluated under a transmission electron microscope (Leo 906, Germany). The renal tissues were studied using electron microscopy to determine the ultrastructural changes of the glomeruli.

Routine immunofluorescence studies (IgG, IgA, IgM, $\mathrm{C} 3$ and C1q) were conducted on the biopsies. Clinical and para-clinical data including blood pressure, blood urea nitrogen (BUN), serum creatinine, hematuria, proteinuria, hearing loss, eye involvement, hypertension, edema, concurrent medical diseases, family history, and other parameters were extracted from hospital files and pathology request sheets.

The final diagnosis (in correlation with clinical presentation) of Alport syndrome was made via electron microscopy appearance of alternative thickening, thinning, splitting, and lamination (basket-weave pattern) of the lamina densa of glomerular basement membrane, while the distinction with thin basement membrane disease was made with the criteria of uniform thinning of the glomerular basement membrane $(<200 \mathrm{~nm})(7,8)$. Fabry disease was confirmed with the diagnostic criteria of lamellar round inclusion bodies with onion skin appearance in the cytoplasm of the visceral epithelial cells and mesangial cells of the glomerulus and as well as characteristic inclusion bodies "Zebra bodies".

The value of electron microscopy was assessed and separated into three groups as follows;

1. Necessary; if the final diagnosis of light microscopy was inconsistent with electron microscopy diagnosis.

2. Supportive; If the electron microscopy study did not alter the primary diagnosis, but provided valuable information confirming the primary diagnosis and ruling out other diagnoses.

3. Non-helpful; if the electron microscopy diagnosis was absolutely the same with light microscopy diagnosis; therefore, electron microscopy was not necessary to confirm the diagnosis.

\section{Ethical issues}

The research followed the tenets of the Declaration of Helsinki. The written informed consent was obtained before doing renal biopsies. The study was approved by the Ethics Committee of Shiraz University of Medical Sciences (IR.sums.med.rec.1398.126). Besides, this study 
was extracted from the M.D, thesis of at this University.

\section{Statistical analysis}

Descriptive statistics for qualitative characteristics were reported as number (\%) and for quantitative characteristics as mean \pm SD. Also, statistical analyses were performed using independent $t$ test, chi-square, and Fisher's exact test, as appropriate. All the statistical analysis was performed in SPSS 19.0 and $P<0.05$ was considered as statistically significant.

\section{Results}

Among all 2865 kidney biopsies sent to our electron microscopy unit, the information of $22(0.77 \%)$ patients of hereditary nephritis including $15(0.52 \%)$ Alport syndrome, $5(0.17 \%)$ thin basement membrane disease, and $2(0.07 \%)$ Fabry disease which were diagnosed by electron microscopy were analyzed in the current study. No cases of Nail-Patella syndrome, collagen type III glomerulopathy, and fibronectin glomerulopathy were identified. The mean age of patients was 16.1 9.0 years and most of them were children (68.2\%). 14 out of 22 cases were male (63.6\%). Proteinuria (81.8\%) and hematuria (59.1\%) were common in them and they had not any malignancy. Tubular atrophy and interstitial fibrosis in electron microscopy were reported for 5 (22.7) and $3(13.6 \%)$ of patients, respectively. Besides, the mean number of observed glomerulus was $10.8 \pm 6.1$ and $7.0 \pm 4.5$ in electron and light microscopy, respectively (Table 1 ).

Thin basement membrane disease did not have any major clinical characteristics aside from hematuria and proteinuria, but a portion of Alport syndrome patients had prominent morbidities with 4 of them (26.7\%) having developed end-stage renal disease who were on dialysis. One of the Fabry disease cases had epilepsy and eye involvement.

The role of electron microscopy was essential for the diagnosis of 19 (86.4) cases and also helpful for the other 3(13.6) cases and there was no case for which electron microscopy was non-necessary.

Mesangioproliferative glomerulonephritis and membranous glomerulonephritis both with 4 out of 14 concurrent diagnoses or misdiagnosis cases among Alport syndrome patients were the most prevalent misdiagnosed disease via light microscopy followed by glomerulosclerosis with $2(14.3 \%)$ cases.

Association between the characteristics under the study with age and gender for 15 Alport cases are reported in Table 2. Although no significant association was detected, this may be due to the small number of sample size. For example, the mean number of the glomerulus in immunofluorescence microscopy was $7.6 \pm 4.8$ for children and $3.4 \pm 1.5$ among adults $(P=0.09)$. Children also had a lower level of mean blood pressure than adults (96.5 versus $107, P=0.19)$. On the other hand, the proportion of hematuria among males was higher than females $(77.8 \%$ versus $33.3 \%, P=0.14$ ) and the level of BUN in females was higher than males (50.2 versus $16.2, P=0.19)$. The proportion of eye involvement was higher in adults than children $(60.0 \%$ versus $20.0 \%)$ and in males than females (44.4\% versus $16.7 \%)$. Hearing loss was higher in children versus adults (5 versus 3 ) and it was higher in adult males versus females (5 versus 3 ).

Eye involvement was detected in $6(27.3 \%)$ patients, and $8(36.4 \%)$ patients had hearing loss. As shown in Table 3 , there was no association between eye involvement or hearing loss status with clinical characteristics such as edema, hematuria, an increase in BUN and creatinine, flank pain and proteinuria. However, the proportion of patients with hypertension was significantly higher among patients with hearing loss problem than others $(62.5 \%$ versus $7.1 \%, P=0.01)$. The relationship between hearing loss and hematuria was in the borderline $(P=0.07)$.

The diagnostic findings of electron microscopy in

Table 1. Demographic, clinical, laboratory and microscopic characteristics of different types of hereditary nephritis

\begin{tabular}{|c|c|c|c|c|}
\hline Variables & Alport syndrome & Thin basement membrane disease & Fabry disease & Total \\
\hline Edema & $7(46.7)$ & $0(0)$ & $2(100)$ & 9 (40.9) \\
\hline Hematuria & $9(60.0)$ & $3(60.0)$ & $1(50)$ & $13(59.1)$ \\
\hline Proteinuria & $13(86.7)$ & $3(60.0)$ & $2(100)$ & $18(81.8)$ \\
\hline Hypertension & $6(40.0)$ & $0(0)$ & $0(0)$ & $6(27.3)$ \\
\hline Eye involvement & $5(33.3)$ & $0(0)$ & $1(50.0)$ & $6(27.3)$ \\
\hline Hearing loss & $8(53.3)$ & $0(0)$ & $0(0)$ & $8(36.4)$ \\
\hline BUN increased & $3(20.0)$ & $1(20.0)$ & $0(0)$ & $4(18.2)$ \\
\hline Creatinine increased & $4(26.7)$ & $1(20.0)$ & $0(0)$ & $5(22.7)$ \\
\hline Systole & $131.7 \pm 23.2$ & $103.3 \pm 15.3$ & $120.0 \pm 14.1$ & $125.3 \pm 23.1$ \\
\hline Diastole & $84.2 \pm 13.1$ & $63.3 \pm 5.8$ & $75.0 \pm 7.1$ & $79.4 \pm 13.9$ \\
\hline Family history of renal disease & $4(26.7)$ & $2(40.0)$ & $0(0)$ & $6(27.3)$ \\
\hline No. of glomerulus in light microscopy & $9.6 \pm 6.6$ & $15.2 \pm 4.1$ & $8.5 \pm 3.5$ & $10.8 \pm 6.1$ \\
\hline No. of the glomerulus in electron microscopy & $6.6 \pm 4.5$ & $7.6 \pm 4.2$ & $7.5 \pm 7.8$ & $7.0 \pm 4.6$ \\
\hline No. of the glomerulus in Immunofluorescence & $6.6 \pm 3.8$ & $6.7 \pm 6.1$ & $2.0 \pm 0.0$ & $5.8 \pm 4.2$ \\
\hline
\end{tabular}

BUN, blood urea nitrogen. 
Table 2. The association between clinical, laboratory and microscopic characteristics with age and sex among patients with Alport syndrome

\begin{tabular}{|c|c|c|c|c|c|c|c|c|}
\hline \multicolumn{9}{|c|}{ Alport syndrome } \\
\hline \multirow[b]{2}{*}{ Variables } & \multicolumn{4}{|c|}{ Age } & \multicolumn{4}{|c|}{ Sex } \\
\hline & $\begin{array}{l}\text { Children } \\
(n=10)\end{array}$ & $\begin{array}{l}\text { Adult } \\
(n=5)\end{array}$ & $\begin{array}{c}\text { Total } \\
(n=15)\end{array}$ & $P$ value & $\begin{array}{l}\text { Male } \\
(n=9)\end{array}$ & $\begin{array}{c}\text { Female } \\
(n=6)\end{array}$ & $\begin{array}{c}\text { Total } \\
(n=15)\end{array}$ & $P$ value \\
\hline Edema & $5(50.0)$ & $2(40.0)$ & $7(46.7)$ & 0.99 & $3(33.3)$ & $4(66.7)$ & $7(46.7)$ & 0.32 \\
\hline Hematuria & $5(50.0)$ & $4(80.0)$ & $9(60.0)$ & 0.58 & $7(77.8)$ & $2(33.3)$ & $9(60.0)$ & 0.14 \\
\hline Proteinuria & $8(80.0)$ & $5(100)$ & $13(86.7)$ & 0.52 & $7(77.8)$ & $6(100)$ & $13(86.7)$ & 0.49 \\
\hline Flank pain & $0(0)$ & $0(0)$ & $0(0)$ & --- & $0(0)$ & $0(0)$ & $0(0)$ & - \\
\hline HTN & $4(40.0)$ & $2(40.0)$ & $6(40.0)$ & 0.99 & $3(33.3)$ & $3(50.0)$ & $6(40.0)$ & 0.62 \\
\hline Eye involvement & $2(20.0)$ & $3(60.0)$ & $5(33.3)$ & 0.25 & $4(44.4)$ & $1(16.7)$ & $5(33.3)$ & 0.58 \\
\hline Hearing loss & $5(50.0)$ & $3(60.0)$ & $8(53.3)$ & 0.99 & $5(55.6)$ & $3(50.0)$ & $8(53.3)$ & 0.99 \\
\hline \multicolumn{9}{|l|}{ Tubular atrophy in EM } \\
\hline No & $8(80.0)$ & $4(80.0)$ & $12(80.0)$ & \multirow{2}{*}{0.99} & $8(88.9)$ & $4(66.7)$ & $12(80.0)$ & \multirow{2}{*}{0.53} \\
\hline Mild & $2(20.0)$ & $1(20.0)$ & $3(20.0)$ & & $1(11.1)$ & $2(33.3)$ & $3(20.0)$ & \\
\hline \multicolumn{9}{|l|}{ Interstitial fibrosis in EM } \\
\hline No & $9(90.0)$ & $4(80.0)$ & $13(86.7)$ & \multirow{2}{*}{0.99} & $8(88.9)$ & $5(83.3)$ & $13(86.7)$ & \multirow{2}{*}{0.99} \\
\hline Mild & $1(10.0)$ & $1(20.0)$ & $2(13.3)$ & & 1 (11.1) & $1(16.7)$ & $2(13.3)$ & \\
\hline BUN & $17.8 \pm 5.6$ & $39.6 \pm 43.5$ & $31.6 \pm 35.6$ & 0.24 & $16.2 \pm 5.3$ & $50.2 \pm 48.3$ & $31.6 \pm 35.6$ & 0.19 \\
\hline $\mathrm{Cr}$ & $1.35 \pm 0.19$ & $1.94 \pm 2.67$ & $1.58 \pm 1.82$ & 0.68 & $1.1 \pm 0.42$ & $2.5 \pm 3.1$ & $1.58 \pm 1.82$ & 0.37 \\
\hline Mean BP & $96.5 \pm 16.1$ & $107.0 \pm 5.8$ & $100 \pm 14.2$ & 0.19 & $100.4 \pm 8.0$ & $99.4 \pm 21.5$ & $100 \pm 14.2$ & 0.91 \\
\hline No. of glomerulus in LM & $6.6 \pm 4.7$ & $7.6 \pm 4.2$ & $7.0 \pm 4.5$ & 0.66 & $7.5 \pm 4.3$ & $6.1 \pm 4.9$ & $7.0 \pm 4.5$ & 0.52 \\
\hline No. of glomerulus in EM & $10.7 \pm 6.9$ & $11.0 \pm 5.0$ & $10.8 \pm 6.1$ & 0.93 & $10.3 \pm 5.7$ & $11.6 \pm 7.1$ & $10.8 \pm 6.1$ & 0.68 \\
\hline No. of glomerulus in IF & $7.6 \pm 4.8$ & $3.4 \pm 1.5$ & $5.8 \pm 4.2$ & 0.09 & $5.3 \pm 3.2$ & $6.3 \pm 5.3$ & $5.8 \pm 4.2$ & 0.70 \\
\hline
\end{tabular}

HTN: hypertension, EM: electron microscopy, LM: light microscopy, IF: immunofluorescence, BUN: blood urea nitrogen, Cr: creatinine, BP: blood pressure.

Table 3. The association of clinicopathologic findings with eye involvement and hearing loss in three hereditary nephritis

\begin{tabular}{|c|c|c|c|c|c|c|}
\hline \multirow[t]{2}{*}{ Variables } & \multicolumn{3}{|c|}{ Eye involvement } & \multicolumn{3}{|c|}{ Hearing loss } \\
\hline & No $(n=16)$ & Yes $(n=6)$ & $P$ value & No $(n=14)$ & Yes $(n=8)$ & $P$ value \\
\hline Edema & $5(31.1)$ & $4(66.7)$ & 0.18 & $4(28.6)$ & $5(62.5)$ & 0.19 \\
\hline Hematuria & $8(50.0)$ & $5(83.3)$ & 0.33 & 6 (42.9) & $7(87.5)$ & 0.07 \\
\hline Proteinuria & $12(75.0)$ & $6(100)$ & 0.54 & $10(71.4)$ & $8(100)$ & 0.25 \\
\hline Flank pain & $0(0)$ & $1(16.7)$ & 0.27 & $1(7.1)$ & $0(0)$ & 0.99 \\
\hline HTN & $4(25.0)$ & $2(33.3)$ & 0.99 & $1(7.1)$ & $5(62.5)$ & 0.01 \\
\hline BUN & $3(18.8)$ & $1(16.7)$ & 0.99 & $2(14.3)$ & $2(25.0)$ & 0.60 \\
\hline $\mathrm{Cr}$ & $3(18.8)$ & $2(33.3)$ & 0.59 & $2(14.3)$ & $3(37.5)$ & 0.31 \\
\hline Tubular atrophy EM & $4(25.0)$ & $1(16.7)$ & 0.99 & $4(28.6)$ & $1(12.5)$ & 0.61 \\
\hline Interstitial Fibrosis EM & $2(12.5)$ & $1(16.7)$ & 0.99 & $2(14.3)$ & $1(12.5)$ & 0.99 \\
\hline
\end{tabular}

HTN: hypertension, EM: electron microscopy, LM: light microscopy, BUN: blood urea nitrogen, Cr: creatinine.

Alport syndrome were alternative areas of thinning and thickening of the glomerular basement membrane with lamellar configuration and splitting changes of the matrix, and semi-thin toluidine blue resin sections demonstrating the same changes that were present in the electron microscopy study (Figure 1). All of the thin basement membrane disease cases had uniform thinning of glomerular basement membrane with thickness of about 180- $200 \mathrm{~nm}$, which was somewhat evident in toluidine blue sections, showing slight thinning of the glomerular basement membrane (Figure 2). The normal glomerular basement membrane thickness is $310-380 \mathrm{~nm}$.
Fabry disease had a characteristic appearance of multiple concentric round lamellae in the mesangial area as well as in the epithelial cells and "zebra bodies". While routine light microscopy studies were normal or non-specific, at best, toluidine blue studies on semi-thin resin blocks, obtained using ultramicrotome, shows numerous round inclusion bodies with onion skin appearance with different sizes in the cytoplasm of parietal, visceral epithelial cells, and mesangial cells (Figure 3).

In some of the cases, interstitial fibrosis and tubular atrophy were observed which was of no diagnostic yield. Some of the light microscopy reports of Alport syndrome 

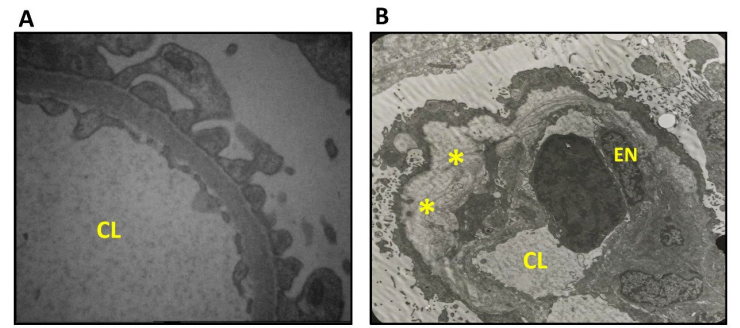

C
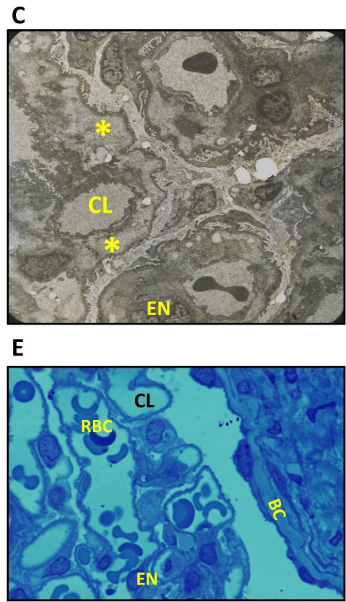

G

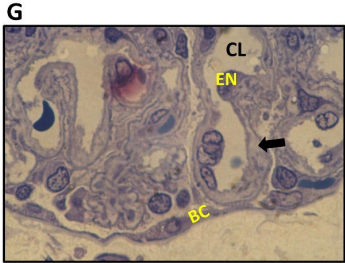

D
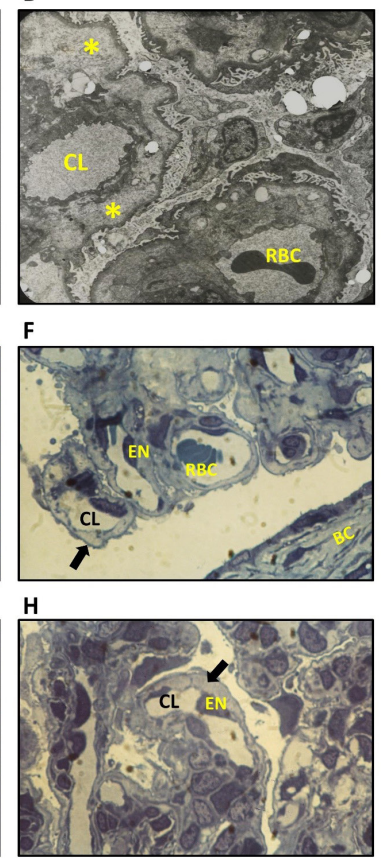

Figure 1. Electron microscopic micrographs of normal glomerulus (A) show uniform normal lamina densa of the basement membrane. In Alport Syndrome (B, C, and D), there are irregular thickening, basket-weave lamellation, and splitting of lamina densa of the basement membrane of the capillary wall (Asterisk). Light microscopic photograph of the semi-thin sections from resin blocks with a 1-micron thickness of normal kidney $(E)$ show a uniform thickness of basement membrane. In Alport Syndrome, irregular thickening and widening of lamina densa appear as pale blue materials instead of the basement membrane of the capillary wall is seen (black arrow) $(F, G$, and $H$ ). (Uranyl acetate and lead citrate; $A \times 2789$, B $\times 2789, \mathrm{C} \times 2156$, and $\mathrm{D} \times 2784$. Toluidine blue; $\mathrm{E}, \mathrm{F}, \mathrm{G}$, and $\mathrm{H} \times 1000$.) $\mathrm{CL}$ : capillary lumen, EN: endothelial cell, $\mathrm{RBC}$ : red blood cell, $\mathrm{BC}$ : Bowman's capsule.

patients showed interstitial foam cells which could be correlated with Alport syndrome.

Electron microscopy micrographs and semi-thin plastic sections stained with toluidine blue of all three hereditary nephritis are illustrated in Figures 1 to 3.

\section{Discussion}

We found that the patients' mean age was $16.1 \pm 9.0$ years and most of them were children. Fourteen out of 22 cases were male $(63.6 \%)$. Proteinuria $(81.8 \%)$ and hematuria $(59.1 \%)$ were the most common findings. Electron microscopy study was essential for reaching a final diagnosis in $86.4 \%$ of the patients and helpful in the remainder $13.6 \%$ of the patients, while there was no case for which electron microscopy was not necessary to
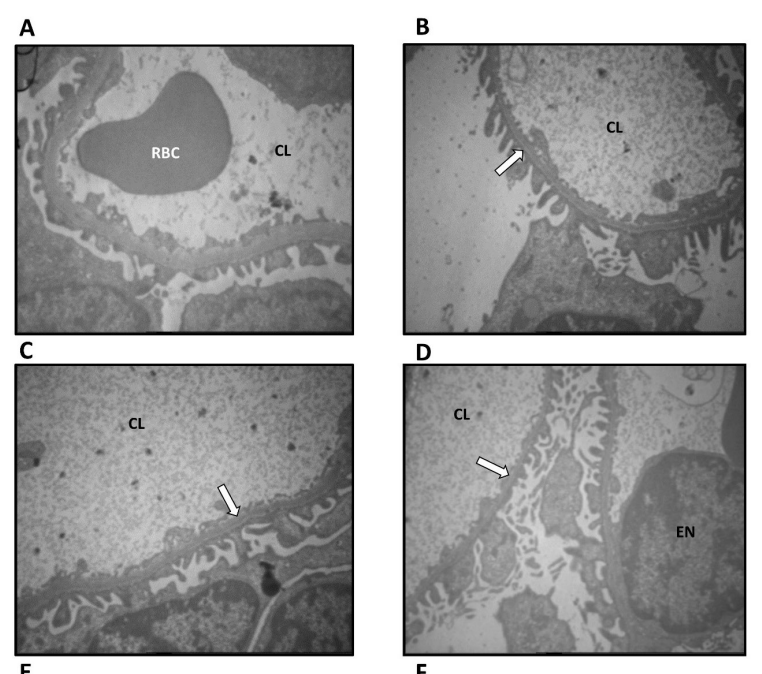

E
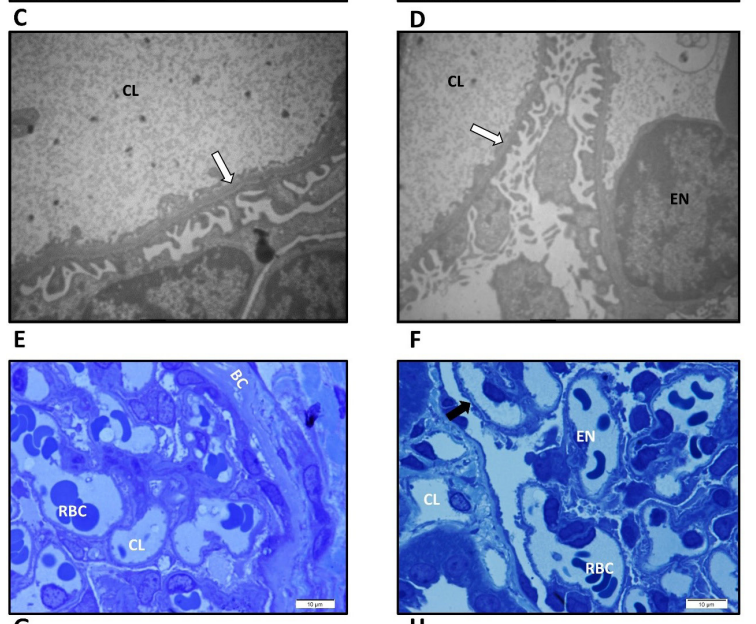

G
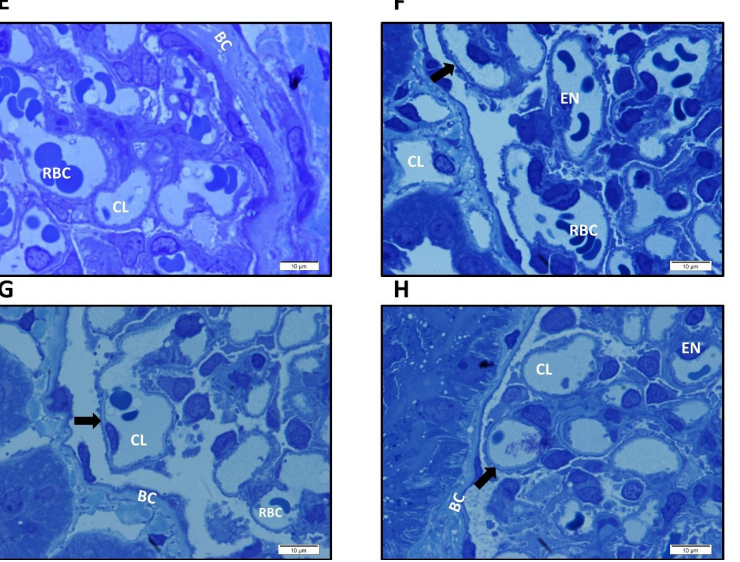

Figure 2. Electron microscopy micrograph of glomerulus capillary wall in the normal kidney $(A)$ shows the normal thickness of the basement membrane (about $350 \mathrm{~nm}$ ). In thin basement membrane disease $(B, C$, and $D)$, there are uniform thinning of the basement membrane of the capillary wall (about $190 \mathrm{~nm}$ ) (white arrow). The foot processes of podocytes are normal. Light microscopic photograph of semi-thin sections from resin blocks with a 1-micron thickness of glomerulus in normal kidney showing the normal thickness of basement membrane of the capillary wall $(E)$. In thin basement membrane disease $(F, G$, and $H)$, slight thinning of the basement membrane of the capillary wall is shown (black arrow). (Uranyl acetate and lead citrate; A, B, C, and D ×3597. Toluidine blue; $\mathrm{E}, \mathrm{F}, \mathrm{G}$, and $\mathrm{H} \times 1000$.)

$\mathrm{CL}$ : capillary lumen, EN: endothelial cell, RBC: red blood cell, BC: Bowman's capsule.

reach the final diagnosis. Eye involvement was detected in $6(27.3 \%)$ patients, and $8(36.4 \%)$ patients had hearing loss. There was no association between eye involvement or hearing loss status with clinical characteristics; while, the proportion of patients with hypertension was higher among patients with hearing loss problem. To the best of our knowledge, this is the first large-scale study on the role of electron microscopy and toluidine blue staining on resin blocks, in addition to a comprehensive clinicopathologic study of hereditary nephritis in Iran to date.

These findings are in the same line with other studies that determined electron microscopy role as necessary for the diagnosis of hereditary nephritis (7,9-19). In all of these studies, routine electron microscopy is recommended to be done for all biopsies and if not possible at least different 
A

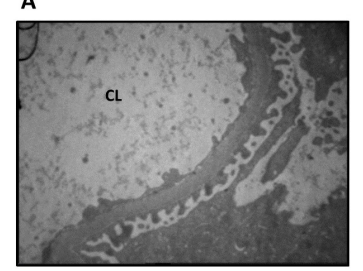

c

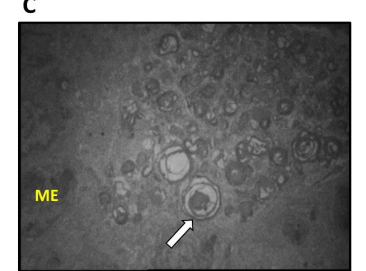

E

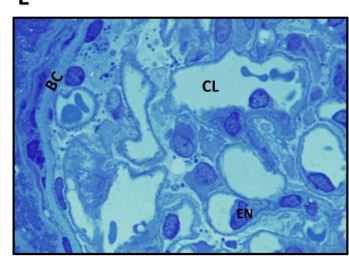

G

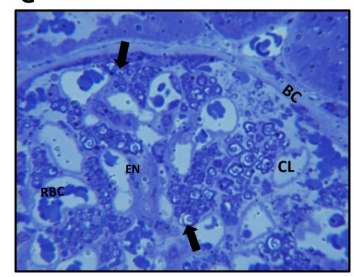

B

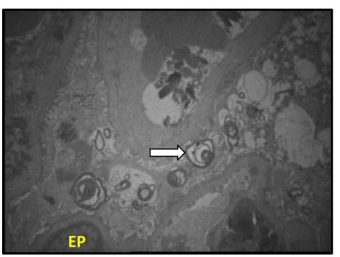

D

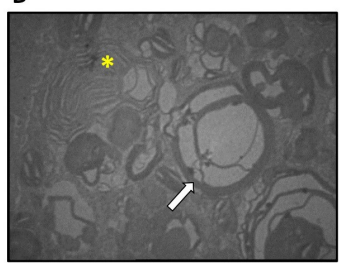

F

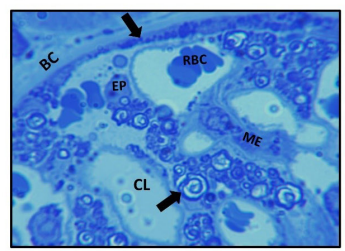

H

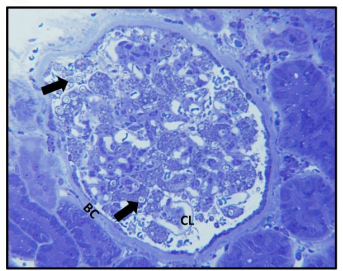

Figure 3. Electron microscopy micrographs of the glomerulus in the normal kidney (A) illustrate normal cytoplasm of the podocyte and mesangial cells. In Fabry Disease (B, C, and D), there is a massive accumulation of laminated round inclusion bodies with onion skin appearance in the cytoplasm of the visceral epithelial cells and mesangial cells of the glomerulus (white arrow). Few inclusion bodies are ovoid with parallel layers (Zebra Bodies) (asterisk). Light Microscopic photograph of semithin sections from resin blocks with a 1-micron thickness of glomerulus in the normal kidney $(E)$ without any inclusion bodies. In Fabry Disease $(F, G$, and $H)$, numerous round bluish inclusion bodies with onion skin appearance with different size (2-7 micron in diameter) are seen in the cytoplasm of parietal, visceral epithelial cells, and mesangial cells (black arrow). (Uranyl acetate and lead citrate; A ×3597, B ×1670, C ×1293, D $\times 3597$. Toluidine blue; $E, F$, and $\mathrm{G} \times 1000, \mathrm{H} \times 400$.)

$\mathrm{CL}$ : capillary lumen, EN: endothelial cell, RBC: red blood cell, $\mathrm{BC}$ : Bowman's capsule, ME: mesangial cell, EP: epithelial cell.

specimens for electron microscopy should be set aside to be used at a later time at the discretion of the pathologist. Although our study did not totally comply with the 100\% necessary rates of electron microscopy in previous studies, it was almost similar because electron microscopy still played a major role in confirming the final diagnosis in the remainder of cases. The study by Kashgarian et al argues that even in the presence of specific anti-collagen type IV immunohistochemistry study, electron microscopy is still crucial for reaching a final diagnosis(10). Additionally, Zuppan et al studied 80 renal biopsies of children for which electron microscopy was essential to diagnosis in $63 \%$ of all cases and helpful in $23 \%$ and it was essential for

$100 \%$ of all the hereditary nephritis cases(16).

In our study, five out of 22 patients had a final diagnosis of thin basement membrane disease; two had a positive family history of hematuria, while one had developed renal failure and three had both hematuria and proteinuria. Three of the thin basement membrane disease cases were initially misdiagnosed as Alport syndrome. Of our five cases of thin basement membrane disease, three showed proteinuria and hematuria compared with a study in which 27 cases of thin basement membrane disease all had hematuria and 21 patients $(77,8 \%)$ had proteinuria (20). A study by Mandache et al included 427 adult patients' kidney biopsy (36 being thin basement membrane disease and 10 Alport syndrome) and demonstrated the coexistence of glomerular diseases and thin basement membrane disease (the type with a mutation in type IV collagen genes, namely col4a3 and col4a3). They concluded that if more specimens are studied by electron microscopy, more thinning of the basement membrane will be detected. Postulating that, if the thinning of glomerular basement membrane is universally and uniformly present, then a thin basement membrane disease will develop. On the other hand, if the modification is sparse and it only shows partial areas of thinning, then it is just a predisposing condition for subsequent glomerulonephritis $(21,22)$.

The total number of hereditary nephritis cases among all patients was relatively low (22 out of 2865 corresponding to $0.07 \%$ ) compared to some large-scale studies, but it is still a large size experiment with regard to local capabilities and past publications. Polito et al reported a hereditary prevalence of $0.7 \%$, out of 9062 biopsies. Zuppan et al had a total of 80 renal biopsies of children, 4 (5\%) of whom were thin basement membrane disease. Bakr et al (Egypt) found in a retrospective study of 1246 native renal biopsies that $8(0.64 \%)$ of them were Alport syndrome. These findings show a heterogeneous compilation of data that is unreliable $(16,23,24)$, probably due to selection bias or maybe we are underdiagnosing these conditions; also, perhaps we would need a more rigorous approach to diagnose these rare conditions.

The clinical manifestations of our Alport syndrome patients were mainly consistent with previous studies. As expected, males (9) were more than females (6) and hematuria, hearing loss, and eye involvement were higher in males, whereas edema, hypertension, and proteinuria were unexpectedly higher in females. According to a study on 148 autosomal recessives (patterns similar to X-linked) Alport syndrome patients, $62 \%$ had endstage renal disease, $64 \%$ had hearing loss, and $17 \%$ had eye involvement. The medium onset of hematuria was 2.5 years, end-stage renal disease 21 while hearing loss was 13 years of age. Of the 49 patients who had electron microscopy, $86 \%$ had typical glomerular basement membrane changes, while $10 \%$ only showed glomerular basement membrane thinning confirming the previous 
argument of misdiagnosis of thin basement membrane disease as Alport syndrome (25). Additionally, in a cohort study, White et al showed that higher proteinuria correlated significantly with glomerulosclerosis and the presence of foam cells. Males have higher morbidity, and also repeated biopsies showed an increase in glomerular basement membrane thickening (26). In a retrospective study on 113 renal biopsies, there were two thin basement membrane disease patients and one Alport syndrome and also one was a Fabry disease patient. Altogether they were diagnosed by electron microscopy (17). Due to the low sample size of our population, statistical tests were mostly insignificant and usually did not show any correlation.

Regarding our two cases of Fabry disease, one had a recent infection, one was on corticosteroids, both had edema and proteinuria, one hematuria, one headache, one photosensitivity, one flank pain, one eye involvement, and one had epilepsy. Renal biopsies had characteristic electron-dense inclusion bodies in the electron microscopy. These findings are in favor of multi-organ involvement consistent with previous studies $(27,28)$. For example, in a case report, one patient who was taking hydroxychloroquine for 14 months presented with subnephrotic proteinuria, showed characteristic Fabry disease deposits in ultrastructural investigation but the deposits had cleared in a subsequent biopsy three years after withdrawal of the drug, suspecting drug-induced renal phospholipidosis. The second case, also presented with sub-nephrotic proteinuria and skin and eye manifestations additionally, which was diagnosed with the help of electron microcopy showing the characteristic "zebra body" deposits. This paper showed, the importance of ultrastructural study in the diagnosis, since clinical investigation should be included in the diagnostic toolkit accordingly to not miss conditions like drug-induced renal phospholipidosis, which might shadow the correct diagnosis with similar presentation (19). In an ongoing cohort study of Fabry disease patients, 6 of whom had done kidney biopsy, all had characteristic inclusion bodies and accumulations in all of the renal cortical ultrastructures, even before the onset of clinical manifestations. Besides, 19\% of the 33 Fabry disease patients had hearing loss, while none had eye involvement in contrast with our study which reports one eye involvement and no hearing loss. Flank pain was a very frequent finding in this study (65\%), which is consistent with our findings(50\%) (28).

\section{Conclusion}

While thin basement membrane disease patients do not usually develop renal failure, prompt diagnosis of Alport syndrome and Fabry disease patients, using electron microscopy, is pertinent to therapeutic decisions, and developing a more comprehensive toolkit for detecting hereditary nephritis is advisable due to a small chance of misdiagnosis even with electron microscopy. We recommend the routine use of toluidine blue study on resin blocks in addition to electron microscopy study for every pathologic workup of kidney biopsies, especially in children.

\section{Limitations of the study}

The key limitation of this study, despite the 16 years of data collection, was its low sample size and sometimes incomplete medical charts which resulted in a lower power for statistical analysis and drawing conclusions. This is mainly due to the rare nature of these diseases and this was already a satisfactory result compared to similar studies with lower numbers. Finally, special collagen type IV immunofluorescence study and the genetic study was not conducted in our research. Therefore, more multicentric studies on larger populations for future research in this area are recommended to validate the repeatability of our results.

\section{Acknowledgments}

The present article was extracted from the medical thesis by Dr. Farshad Dehghani. The authors wish to thank the Research Consultation Center (RCC) at Shiraz University of Medical Sciences for assistance in the English editing of this manuscript. Also, the authors would like to thank Dr. Simin Torabinezhad for reviewing routine light microscopy slides, Ms. Hamide Jabedarbashy (technician of electron microscopy unit) and Dr. Shahriary, Moravej, Kamalinia, Bagheri, Ghasemian, Hafezi Rad, and Zarmahi (medical interns at Shiraz University of Medical Sciences) for their precious help for data gathering from hospital records and pathology reports. The abstract of this paper was presented in the 21 st annual congress and 4th international congress of pathology and laboratory medicine (September 2019, Tehran, Iran. http://www. congress.iranpath.org/fa).

\section{Authors' contribution}

SMO is the first author and responsible for study design, data acquisition, critical revision and final approval of the work. HRS contributed to data analysis, critical revision and final approval of the study. NP contributed to data acquisition, critical review and final approval of the study. SHO contributed to the conception of the work, data acquisition, critical review and final approval of the study. Finally, FD is the corresponding author and was responsible for data acquisition and interpretation, drafting and final approval of this work. All authors read and approved the final paper.

\section{Conflicts of interest}

The authors declare no conflict of interest.

Ethical considerations

Ethical issues (including plagiarism, data fabrication, 
double publication) have been completely observed by the authors.

\section{Funding/Support}

The study was supported by the Deputy of Research and Technology Development at Shiraz University of Medical Science (\#1397-01-01-18942).

\section{References}

1. Gubler MC. Inherited diseases of the glomerular basement membrane. Nat Clin Pract Nephrol. 2008;4:24-37. doi: 10.1038/ncpneph0671.

2. Goldblum JR, Lamps LW, McKenney JK, Myers JL. Rosai and Ackerman's Surgical Pathology. Elsevier; 2017. p. 2142.

3. Kashtan CE, Segal Y. Genetic disorders of glomerular basement membranes. Nephron Clin Pract. 2011;118:c9-18. doi: $10.1159 / 000320876$

4. Nozu K, Nakanishi K, Abe Y, Udagawa T, Okada S, Okamoto $\mathrm{T}$, et al. A review of clinical characteristics and genetic backgrounds in Alport syndrome. Clin Exp Nephrol. 2019;23:158-68. doi: 10.1007/s10157-018-1629-4.

5. Kashtan CE, Michael AF. Alport syndrome. Kidney Int. 1996;50:1445-63. doi: 10.1038/ki.1996.459.

6. Jennette JC, Olson JL, Silva FG, D’Agati VD. Heptinstall's Pathology of the Kidney. 7th ed: Wolters Kluwer; 2014. p. 2898.

7. Haas M. Alport syndrome and thin glomerular basement membrane nephropathy: a practical approach to diagnosis. Arch Pathol Lab Med. 2009;133:224-32. doi: 10.1043/15432165-133.2.224.

8. Dische FE. Measurement of glomerular basement membrane thickness and its application to the diagnosis of thinmembrane nephropathy. Arch Pathol Lab Med. 1992;116:439.

9. Haas M. A reevaluation of routine electron microscopy in the examination of native renal biopsies. J Am Soc Nephrol. 1997;8:70-6.

10. Kashgarian M. The Importance of Electron Microscopy and Immunofluorescence in Evaluation of Renal Biopsies. AJSP Rev Rep. 1998;3:163-8.

11. Sementilli A, Moura LA, Franco MF. The role of electron microscopy for the diagnosis of glomerulopathies. Sao Paulo Med J. 2004;122(3):104-9. doi: 10.1590/s151631802004000300006.

12. Collan $Y$, Hirsimäki $P$, Aho $H$, Wuorela $M$, Sundström $\mathrm{J}$, Tertti R, et al. Value of electron microscopy in kidney biopsy diagnosis. Ultrastruct Pathol. 2005;29:461-8. doi: 10.1080/01913120500323381.

13. Mubarak M, Kazi JI. Role of immunofluorescence and electron microscopy in the evaluation of renal biopsies in nephrotic syndrome in a developing country. Ultrastruct Pathol. 2009;33:260-4. doi: 10.3109/01913120903296952.

14. Elhefnawy NG. Contribution of electron microscopy to the final diagnosis of renal biopsies in Egyptian patients. Pathol Oncol Res. 2011;17:121-5. doi: 10.1007/s12253-010-9290-6.
15. Pearson JM, McWilliam LJ, Coyne JD, Curry A. Value of electron microscopy in diagnosis of renal disease. J Clin Pathol. 1994;47:126-8. doi: 10.1136/jcp.47.2.126.

16. Zuppan C. Role of electron microscopy in the diagnosis of nonneoplastic renal disease in children. Ultrastruct Pathol. 2011;35:240-4. doi: 10.3109/01913123.2011.606394.

17. Wagrowska-Danilewicz M, Danilewicz M. Current position of electron microscopy in the diagnosis of glomerular diseases. Pol J Pathol. 2007;58:87-92.

18. Arslansoyu Camlar S, Ünlü M, Soylu A, Karaca D, Sarioglu S, Kavukcu S. Contribution of electron microscopy to the clinicopathologic diagnosis in childhood glomerular renal diseases. Fetal Pediatr Pathol. 2019;38:299-306. doi: $10.1080 / 15513815.2019 .1587119$.

19. de Menezes Neves PDM, Machado JR, Custódio FB, Dos Reis Monteiro MLG, Iwamoto S, Freire M, et al. Ultrastructural deposits appearing as "zebra bodies" in renal biopsy: Fabry disease? - comparative case reports. BMC Nephrol. 2017;18:157. doi: 10.1186/s12882-017-0571-0.

20. Kajimoto Y, Endo Y, Terasaki M, Kunugi S, Igarashi T, Mii A, et al. Pathologic glomerular characteristics and glomerular basement membrane alterations in biopsy-proven thin basement membrane nephropathy. Clin Exp Nephrol. 2019;23:638-49. doi: 10.1007/s10157-018-01687-1.

21. Mandache E, Gherghiceanu M. Ultrastructural defects of the glomerular basement membranes associated with primary glomerular nephropathies. Ultrastruct Pathol. 2004;28:103-8. doi: 10.1080/01913120490445016.

22. Kfoury H, Arafah M. The pathological spectrum associated with the ultrastructural finding of thin glomerular basement membrane: A tertiary medical city experience and review of the literature. Ultrastruct Pathol. 2017;41:51-4. doi: 10.1080/01913123.2016.1258021.

23. Bakr A, Eid R, Sarhan A, Hammad A, El-Refaey AM, ElMougy A, et al. Fifteen years of kidney biopsies in children: a single center in Egypt. Saudi J Kidney Dis Transpl. 2014;25:1321-7.

24. Polito MG, de Moura LA, Kirsztajn GM. An overview on frequency of renal biopsy diagnosis in Brazil: clinical and pathological patterns based on 9,617 native kidney biopsies. Nephrol Dial Transplant. 2010;25:490-6. doi: 10.1093/ndt/ gfp355.

25. Lee JM, Nozu K, Choi DE, Kang HG, Ha IS, Cheong HI. Features of autosomal recessive Alport syndrome: a systematic review. J Clin Med. 2019;8:E178. doi: 10.3390/jcm8020178.

26. White RH, Raafat F, Milford DV, Komianou F, Moghal NE. The Alport nephropathy: clinicopathological correlations. Pediatr Nephrol. 2005;20:897-903. doi: 10.1007/s00467-0051955-0.

27. Zarate YA, Hopkin RJ. Fabry's disease. Lancet. 2008 8;372:1427-35. doi: 10.1016/S0140-6736(08)61589-5.

28. Wijburg FA, Bénichou B, Bichet DG, Clarke LA, Dostalova G, Fainboim A, et al. Characterization of early disease status in treatment-naive male paediatric patients with Fabry disease enrolled in a randomized clinical trial. PLoS One. 2015;10:e0124987. doi: 10.1371/journal.pone.0124987.

Copyright $\odot 2020$ The Author(s); Published by Nickan Research Institute. This is an open-access article distributed under the terms of the Creative Commons Attribution License (http://creativecommons.org/licenses/by/4.0), which permits unrestricted use, distribution, and reproduction in any medium, provided the original work is properly cited. 